\title{
The longitudinal associations between change in physical activity and cognitive functioning in older adults with chronic illness (es)
}

Esmee Volders $^{1 *}$, Renate H. M. de Groot ${ }^{2,3}$, Catherine A. W. Bolman ${ }^{1}$ and Lilian Lechner ${ }^{1}$

\begin{abstract}
Background: Regular physical activity (PA) is potentially beneficial for age-related cognitive decline. Although moderate-to-vigorous physical activity (MVPA) is mostly advised, older adults with chronic illnesses might benefit more from light physical activity (LPA), as they suffer from mobility problems, pain, and fatigue, limiting highintensity PA. Therefore, the longitudinal association between change in LPA and MVPA and the change in cognitive functioning (CF) is investigated in older adults with chronic illnesses.

Methods: In total 432 older adults (mean age 73.7 [ \pm 6.1 ] years; $46.8 \%$ female) with at least one chronic illness participated in this longitudinal observational study. Longitudinal associations between accelerometer-assessed change in PA (LPA and MVPA) and change in CF, measured with an objective validated neuropsychological test battery, were tested with multivariate linear regressions.

Results: An increase in LPA between baseline and 6 months follow-up was significantly associated with improved short-term verbal memory and inhibition over the first 6 months. In addition, the change score in LPA over the first 6 months was predictive for the change score in short-term verbal memory over 12 months. Furthermore, an increase in MVPA between baseline and 6 months follow-up was significantly associated with a decrease in longerterm verbal memory scores over the same six-month period.

Conclusions: For older adults with chronic illnesses who may experience difficulties in being sufficiently active, an increase in LPA is probably more achievable than an increase in MVPA. In addition, an increase in LPA enhances CF more than an increase in MVPA does.
\end{abstract}

Trial registration: Netherlands Trial Register NL6005; Date of Registration 21-03-2017.

Keywords: Cognition, Physical activity, Ageing, Chronic illness, Older adults

\footnotetext{
* Correspondence: esmee.volders@ou.nl

${ }^{1}$ Faculty of Psychology, Open University of the Netherlands, 6419 AT Heerlen, the Netherlands

Full list of author information is available at the end of the article
}

C C The Author(s). 2021 Open Access This article is licensed under a Creative Commons Attribution 4.0 International License, which permits use, sharing, adaptation, distribution and reproduction in any medium or format, as long as you give appropriate credit to the original author(s) and the source, provide a link to the Creative Commons licence, and indicate if changes were made. The images or other third party material in this article are included in the article's Creative Commons licence, unless indicated otherwise in a credit line to the material. If material is not included in the article's Creative Commons licence and your intended use is not permitted by statutory regulation or exceeds the permitted use, you will need to obtain permission directly from the copyright holder. To view a copy of this licence, visit http://creativecommons.org/licenses/by/4.0/. The Creative Commons Public Domain Dedication waiver (http://creativecommons.org/publicdomain/zero/1.0/) applies to the data made available in this article, unless otherwise stated in a credit line to the data. 


\section{Background}

Cognitive decline can impair the quality of life of older adults and reduce their independence [1]. Older adults with chronic illnesses are especially prone to have lower levels of cognitive functioning (CF) compared to healthy older adults [2]. Regular physical activity (PA) has been argued as an important protective factor against agerelated cognitive decline, with PA at a moderate-tovigorous intensity mostly being advised [3-5]. However, older adults with chronic illnesses, such as with osteoarthritis and cardiovascular diseases, may face difficulties in being sufficiently active due to mobility problems, pain, and fatigue [6]. Therefore, performing activities at a lighter intensity is more achievable for older adults with chronic illnesses. However, only few studies have investigated the relationship between light physical activity (LPA) and CF [7-9], and the study by Stubbs et al. [9] was the only one researching the longitudinal association. Hence, the longitudinal association in older adults with chronic illnesses between change in both LPA and moderate-to-vigorous physical activity (MVPA), on the one hand and the change in $\mathrm{CF}$, on the other hand is investigated.

PA can be assessed subjectively with self-report questionnaires or objectively with accelerometers, and it can be categorised into different intensity levels: sedentary, low, moderate, and vigorous [10]. Examples of LPA activities are walking at a low speed and light household chores. Bicycling at a low speed, vacuuming, and walking briskly are examples of moderate-intensity PA. Running, carrying heavy loads, and swimming laps are examples of vigorous-intensity PA [11]. The effects of PA on physical health outcomes can be different at different intensity levels. Up to a decade ago, most research investigating the physical health benefits of PA relied mainly on selfreported PA and often did not make a distinction between PA intensities, nor was LPA considered [12]. However, people's ability to recall PA of moderate-to-vigorous intensity is much more accurate than that of light intensity [10]. Currently, guidelines prescribe at least $150 \mathrm{~min}$ of MVPA spread over preferably multiple days per week to achieve physical health benefits $[13,14]$, such as lower risk for obesity, cardiovascular disease, some types of cancer, osteoporosis, and premature death, while mostly overlooking the role of LPA [15]. However, more recent evidence from studies assessing PA with accelerometers proved that LPA can have physical health benefits too $[12,16]$. These studies suggest that LPA is inversely associated with allcause mortality risk and associated favourably with some cardio-metabolic risk factors, including waist circumference, triglyceride levels, insulin, and presence of metabolic syndrome.

Next to the physical health benefits of PA, the evidence for cognitive health benefits of PA grows. PA can promote cognitive brain health, defined by the US Centres for Disease Control and Prevention [17] as an ability to perform all the mental processes of cognition, including the ability to learn and judge, use language, and remember, and it can counteract many effects of cognitive ageing $[3,5,18]$. The association between PA and CF has been confirmed in both cross-sectional and longitudinal cohort studies $[19,20]$. However, evidence from studies regarding the effect of PA interventions on CF in older adults is inconsistent [21]. Some meta-analyses have found moderate cognitive improvements as a result of PA interventions in older adults [4, 18, 22, 23]. Yet, other meta-analyses demonstrated little to no cognitive improvements [24, 25], even when the intervention led to objectively measured increased fitness and PA behaviour [26]. Almost all meta-analyses included studies that did not take into account the actual effect of the PA intervention on PA behaviour in daily life or cardiorespiratory fitness. Thus, besides the wide variety in interventions (different types of PA activities, duration and frequency of the sessions, and the duration of the programme), one of the possible explanations for this discrepancy can be found in the many different ways in which PA was taken into account in these studies [27].

In line with research into the physical health benefits of PA, research into the cognitive health benefits of PA have also mainly focused on MVPA. When looking at effects of MVPA on CF, the executive functions (inhibition, shifting, and updating) seem to benefit the most $[18,23]$. Executive functions are higher-order cognitive processes that are necessary to control cognitive behaviour. Nonetheless, studies so far have focused less on the relation of PA on a lower intensity level with $\mathrm{CF}$, but it appears that LPA could also be beneficial for CF [2830]. In recent studies, LPA has been positively associated with shifting, word fluency, processing speed, and a reduced rate of cognitive ability decline [7-9]. However, until now there is little information on whether LPA influences different aspects of CF than MVPA does.

Despite the promising benefits of PA as described above, older adults are the least physically active age group, especially when they suffer from chronic illnesses [31, 32]. Fatigue and pain are examples of PA-related barriers experienced by older adults with chronic illnesses that may result in these low levels of PA $[6,31]$. Increasing PA in general, especially through MVPA, is often difficult, and it is sometimes accompanied by risks of injury and deterioration because of physical complications. Furthermore, increasing LPA is probably easier and safer for older adults. Therefore, it would be justified to determine which intensities of PA are associated with CF. Taking into account the fact that LPA (i.e., light housework, slow walking) is the dominant type of PA in older adults, especially in those who suffer from 
chronic illnesses, and that few of these older adults participate in meaningful amounts of MVPA, it is crucial to determine how changes in both LPA and MVPA are related to change in $\mathrm{CF}$.

Randomised controlled trials are one of the best methods to test intervention effects in general and, more specifically, the effects of PA on CF. In a previous study, we tested the cognitive effects of a computer-tailored PA stimulating intervention, which consisted of three times personalised PA advice within 4 months delivered by mail and online, for older adults with chronic illnesses. We hypothesised that increasing PA would lead to improved CF. However, no intervention effects on CF were found six and 12 months after baseline [33]. The most likely explanation for this null finding was that the intervention did not lead to more objectively measured PA in this population [34].

Even though our intervention had limited effects on PA behaviour and no effects on CF, it is relevant and scientifically valuable to investigate whether and how the change in PA, operationalised as MVPA as well as LPA, is related to a change in CF in older adults with chronic illnesses, independent of the intervention. A powerful aspect of the current study is the objective measurement of PA by accelerometers on different time points and thereby taking LPA into account. We hypothesise that participants who increased their PA (i.e., between baseline and 6 months follow-up, between 6 months follow-up and 12 months follow-up, and between baseline and 12 months follow-up) showed more progress on the CF tests than those who did not increase their PA. Furthermore, we hypothesise that associations between change in PA and change in CF are expected to be stronger when considering similar time periods, as stated above, in comparison to the consideration of different, non-parallel time periods (change in PA between baseline and 6 months follow-up in relation to change in CF between baseline and 12 months follow-up). The main argument behind this hypothesis is that potential associations can fade away over time. However, it can take some time to establish a lasting change in CF. It is presumed that these changes are due to improved vascularisation, facilitation of synaptogenesis, decreased systemic inflammation, and reduced disordered protein deposition, and these lasting changes do not take place over-night [35]. Because different aspects of CF can respond differently to PA $[18,29]$, we analyse the associations between change in PA (LPA and MVPA) and change in different aspects of CF. The selected aspects of CF are verbal memory, shifting, inhibition, and information processing because these functions are known to deteriorate with age and can possibly improve with increased PA [19, 26, 36-39].

\section{Methods}

\section{Study design and population}

The present study on the association of changes in LPA and MVPA over six and 12 months, respectively, with changes in CF outcomes over the same period was part of the Active Plus and Cognitive Functioning project [40]. This project concerned a clustered two-group randomised controlled trial with a waiting list control group with assessments at baseline, 6 months, and 12 months, focused on the effect of the Active Plus intervention on CF. Intervention group participants received three times computer-tailored PA stimulating advice within 4 months (i.e., at baseline, after 2 months, and after 3 to 4 months). The online- and print-delivered advice were tailored to the specific needs and wishes of the participant and focused on incorporating PA in daily life. Data of all participants (both intervention group and control group) who completed the randomised controlled trial were used in the present study. Ethical approval for the study was obtained from the Research Ethics Committee (cETO) of the Open University, and the trial is registered in the Dutch Trial Register (protocol no. NL6005). An elaborate explanation of the study protocol was published elsewhere [40].

Six hundred and twenty-three participants were recruited from seven municipalities, which randomly invited between 500 and 4000 independently living adults aged 65 years or older living in a specific neighbourhood through an invitation letter via post. The participants met the following criteria (checked by a self-report questionnaire and a phone call with the researcher): 1) aged 65 years or older; 2) fluent in the Dutch language; 3 ) suffering from at least one self-reported chronic illness that affects mobility (e.g., chronic obstructive pulmonary disease, osteoarthritis, chronic heart disease) or other physical problems (e.g., visually or hearing impaired) that may affect mobility; 4) no self-reported severe cognitive problems; and 5) no wheelchair use. All participants provided written informed consent.

\section{Procedure}

At baseline and at six and 12 months, the following procedure was adhered to: PA was assessed with an accelerometer (ActiGraph GT3X-BT) placed on the participants' right hip for seven consecutive days prior to the CF tests. The CF tests were conducted by a trained researcher or student at the participants' home. Inquisit 5 software [41] was used on a tablet (iPad Air 2) to execute the CF tests. The CF tests started with the first part of the Verbal Learning Test (VLT), followed by the Trail Making Test (TMT) parts A and B, the Stop-Signal Task (SST), the Letter Digit Substitution Test (LDST), and the second part of the VLT. After completing the CF tests, participants received a questionnaire to fill out within 2 weeks. The 
Table 1 Outcome measures ${ }^{b}$

\begin{tabular}{|c|c|c|c|c|c|c|}
\hline $\begin{array}{l}\text { Measurement } \\
\text { Instrument }\end{array}$ & Concept & Measure & Scoring/ missing items & $\begin{array}{l}\text { Scoring } \\
\text { range }\end{array}$ & $\begin{array}{l}\text { Higher } \\
\text { score } \\
\text { indicates }\end{array}$ & $\begin{array}{l}\% \\
\text { valid } \\
\text { a }\end{array}$ \\
\hline \multicolumn{7}{|c|}{ Primary outcome measures } \\
\hline \multirow[t]{3}{*}{ VLT } & $\begin{array}{l}\text { Verbal } \\
\text { memory }\end{array}$ & Learning curve ratio & $\begin{array}{l}(\text { Trial } 1+(\text { Trial } 2 \text {-Trial } 1)+(\text { Trial 3-Trial 2) }+(\text { Trial 4-Trial } \\
3)+(\text { Trial } 5 \text {-Trial 4) }) / 5\end{array}$ & $\begin{array}{l}0-3 \\
\text { words } \\
\text { per trial }\end{array}$ & $\begin{array}{l}\text { Better } \\
\text { learning } \\
\text { capacity }\end{array}$ & $98 \%$ \\
\hline & & $\begin{array}{l}\text { Mean number of } \\
\text { recalled words trial 1-5 }\end{array}$ & $($ Trial $1+$ Trial $2+$ Trial $3+$ Trial $4+$ Trial 5) $/ 5$ & $\begin{array}{l}0-15 \\
\text { words }\end{array}$ & $\begin{array}{l}\text { Better short- } \\
\text { term verbal } \\
\text { memory }\end{array}$ & \\
\hline & & $\begin{array}{l}\text { Number of words } \\
\text { recalled in delayed trial }\end{array}$ & & $\begin{array}{l}0-15 \\
\text { words }\end{array}$ & $\begin{array}{l}\text { Better long- } \\
\text { term verbal } \\
\text { memory }\end{array}$ & \\
\hline TMT & $\begin{array}{l}\text { Task } \\
\text { switching }\end{array}$ & $\begin{array}{l}\text { Time to complete part } \\
\mathrm{B} \text { minus time to } \\
\text { complete } \mathrm{A} \text { in sec }\end{array}$ & & $0-\infty$ sec & $\begin{array}{l}\text { Worse } \\
\text { shifting } \\
\text { capacity }\end{array}$ & $96 \%$ \\
\hline SST & Inhibition & SSRT in ms & $\begin{array}{l}\text { The SSRT is estimated in accordance with De Jong et al. } \\
\text { [42] and Tannock et al. [43]. Negative SSRT values are } \\
\text { excluded from the analyses [44]. }\end{array}$ & $\begin{array}{l}0-1500 \\
\text { ms }\end{array}$ & $\begin{array}{l}\text { Worse } \\
\text { inhibition }\end{array}$ & $90 \%$ \\
\hline LDST & $\begin{array}{l}\text { Processing } \\
\text { speed }\end{array}$ & $\begin{array}{l}\text { Number of correct } \\
\text { substitutions }\end{array}$ & & $\begin{array}{l}0-125 \\
\text { subs }\end{array}$ & $\begin{array}{l}\text { Better } \\
\text { processing } \\
\text { speed }\end{array}$ & $96 \%$ \\
\hline \multicolumn{7}{|l|}{ Physical activity } \\
\hline \multirow[t]{2}{*}{$\begin{array}{l}\text { ActiGraph } \\
\text { GT3X-BT }\end{array}$} & PA & $\begin{array}{l}\text { MVPA minutes per } \\
\text { week }\end{array}$ & $\begin{array}{l}\text { Data downloaded with frequency extension on with Actilife } \\
\text { software [45]. Valid if worn } 4 \text { days during } 10 \mathrm{~h} \text { or more [46]. } \\
\text { Non-wear definition by algorithm of Choi et al. [47]. PA scor- } \\
\text { ing by Freedson-VM cut-off points [48] and by Aguilar-Fariaz } \\
\text { cut-off points [49]. }\end{array}$ & $\begin{array}{l}0-6720 \\
\min \end{array}$ & More MVPA & $96 \%$ \\
\hline & & LPA minutes per week & & $\begin{array}{l}0- \\
10,080 \\
\min \end{array}$ & More LPA & \\
\hline
\end{tabular}

Abbreviations: VLT Verbal learning test, TMT Trail making test, SST Stop-signal task, LDST Letter digit substitution test, PA Physical activity, SSRT Stop-signal reaction time, MVPA Moderate-to-vigorous physical activit, LPA Light physical activity. ${ }^{\text {a }}$ Test outcomes were excluded if scores were deemed invalid by test administer when 1) technical problems occurred, 2) participants refused to complete a test or lacked motivation, 3) participants had physical limitations (arm amputated, hearing loss, etc.) or cognitive restrictions (participant is unable to understand the instruction), or 4) participants deviated from the instructions. ${ }^{b}$ Derived from Table 1 in [33]

questionnaires were used to gather information on demographic variables, but also on concepts that are outside the scope of this study (e.g., self-reported PA, self-reliance, health-related quality of life). The four-month intervention started directly after completing the baseline measurement.

\section{Outcome measures}

Cognitive functioning Table 1 provides an overview of all outcome measures. The aspects of CF (e.g., verbal memory, task switching, inhibition, processing speed) assessed in this study are chosen because they are known to deteriorate with age and can possibly improve with increased PA (Table 1) [19, 26, 36-40].

In the VLT [37, 50], which assesses verbal memory, 15 common monosyllabic words representing concrete objects were presented one by one on an iPad screen in fixed order, with a presentation time of $1 \mathrm{~s}$ and an interstimulus interval of $1 \mathrm{~s}$. Afterwards, participants were asked to verbally recall the words they had remembered. The first trial was followed by four more trials in which the words were presented in identical order and each followed by an immediate free recall procedure. After a delay of 15-25 min in which the remaining CF tests were assessed, and unexpectedly for the participants, the instruction was given to recall the 15 words learned once more. Finally, a recognition trial was administered where participants had to recognise the 15 learned words out of 30 words. Outcome measures for the VLT were the learning curve ratio over trials $1-5$, the mean number of recalled words in trial $1-5$, and the number of words recalled in the delayed trial (Table 1 ).

During the TMT parts A and B [51], which can be used to assess task switching when both parts are administered, participants had to draw lines with their fingers on an iPad screen connecting 25 randomly placed numbers in the correct order (part A) or numbers and letters alternatively (part B). Both parts A and B were preceded by a practice trial. The time in seconds 
required to complete the task was noted for each task. The outcome measure task switching was operationalised as the time to complete part B minus the time to complete part A [52].

In the SST [53], which is an inhibition task, participants had to quickly press the left-hand button if the arrow on the iPad screen pointed to the left and press the right-hand button if the arrow pointed to the right. However, when a signal beep was played after the presentation of the arrow, participants had to inhibit their reaction and withheld from pressing either of the buttons. These beeps occurred in $25 \%$ of the trials. Firstly, participants could practice the task in a block of 32 trials. Afterward, three blocks of 64 trials were completed with $10 \mathrm{~s}$ of rest in between blocks. The stop-signal delay between presentation of the arrow and signal beep was varied and depended on participants' performance. The delay, which started at 250 milliseconds (ms), was increased by $50 \mathrm{~ms}$ if the previous inhibition was successful. The delay got shorter by $50 \mathrm{~ms}$ if the previous inhibition was unsuccessful. This stop-signal delay staircase design ensured that participants were able to inhibit their response on approximately half of all trials. The inhibition outcome measure was operationalised as the stop-signal reaction time in ms (SSRT).

During the LDST [38], which is a processing speed task, participants were presented with a matrix. Odd rows contained letters; even rows contained empty answer boxes. The task was to translate the letters by clicking the corresponding digits with the help of a provided key. After a practice round of 10 letters, the participant had $60 \mathrm{~s}$ to replace as many randomised letters with the appropriate digit indicated by the key. The outcome measure for the LDST was the number of correct substitutions made in $60 \mathrm{~s}$.

Physical activity PA was objectively measured using the ActiGraph GT3X-BT (ActiGraph, Pensacola, FL, USA). The accelerometer was placed on the right hip with an elastic belt. Participants were asked to wear the accelerometer for seven consecutive days. However, participants were not obliged to wear the device during the night. While showering or swimming, the accelerometer had to be removed.

Demographic and health characteristics Demographics and health characteristics were part of the Active Plus and Cognitive Functioning project [40]. As age, gender, educational level, marital status (living together with a spouse or living single), body mass index (BMI), and physical impairment are known to influence PA [54] and some also CF [55], these factors, assessed at baseline, were taken into account in the current study. Educational level was categorised into low (i.e., primary, basic vocational, or lower general school), moderate (i.e., medium vocational school, higher general secondary education, and preparatory academic education), or high (i.e., higher vocational school or university level) according to the Dutch educational system.

BMI was defined as the body mass (in $\mathrm{kg}$ ) divided by the square of body height (in $\mathrm{m}$ ). The degree of physical impairment was measured with a self-report questionnaire [56]. The participant stated for 14 common chronic illnesses (e.g., cardiovascular, osteoarthritis) and physical conditions (e.g., hearing or visually impaired) to what degree he/she was limited in his/her PA by one of the illnesses mentioned or by another illness not mentioned. For each chronic illness, the participant scored the degree of impairment on a five-point scale $(0=$ not applicable, $1=$ not at all/hardly, $2=$ a little, $3=$ very, $4=$ extremely). Consequently, degree of impairment was computed into three categories based on the following rules: 1) little impaired: a maximum score of 1 on at least one question; 2) medium impaired: a maximum score of 2 on at least one question; 3) very impaired: at least a score of 3 or 4 on at least one question.

Statistical analyses Baseline characteristics are described for all participants who finished the randomised controlled trial using means and standard deviations for normally distributed continuous variables, medians and inter-quartile differences for non-normally distributed continuous variables, and frequency and percent for categorical variables. For further analyses, we log transformed the non-normally distributed TMT outcome measure. To assess predictors of dropout at six and 12 months, logistic regression with baseline outcome measures, demographics, and degree of impairment regarding chronic illnesses was performed and odds ratios (OR) are noted.

We tested the following longitudinal associations between PA and CF using multivariate linear regressions with the CF outcome at six or 12 months as the dependent variable and the change in PA as the independent variable (Fig. 1): 1) associations between change in PA over the first 6 months and change in CF outcomes over the same period; 2) associations between change in PA between 6 months and 12 months followup and change in CF outcomes over the same period; 3 ) associations between change in PA between baseline and 12 months follow-up and change in CF outcomes over the same period; and 4) associations of the predictive value of change in PA between baseline and 6 months follow-up for change in CF outcomes between baseline and 12 months follow-up.

Change in LPA and MVPA was calculated by subtracting the former PA score from the latter follow-up score only if scores at both time points were known. 


\begin{tabular}{|c|c|c|c|c|}
\hline Hypothesis & $\begin{array}{l}\text { Outcome } \\
\text { measure }\end{array}$ & Baseline & $\begin{array}{l}6 \\
\text { months }\end{array}$ & $\begin{array}{l}12 \\
\text { months }\end{array}$ \\
\hline \multirow[t]{2}{*}{1} & PA & & & \\
\hline & $\mathrm{CF}$ & & & \\
\hline \multirow[t]{2}{*}{2} & PA & & & \\
\hline & $\mathrm{CF}$ & & & \\
\hline \multirow[t]{2}{*}{3} & PA & & & \\
\hline & $\mathrm{CF}$ & & & \\
\hline \multirow[t]{2}{*}{4} & PA & & & \\
\hline & $\mathrm{CF}$ & & & \\
\hline
\end{tabular}

Fig. 1 Overview of tested hypotheses. Associations between change in PA (independent variable) and change in CF (dependent variable) are tested using the data from time-points presented in colour

Otherwise, these scores were not taken into account in the analyses. Only valid CF tests were included in the analyses. The regressions were adjusted for age, gender, educational level, marital status, BMI, degree of impairment, baseline or 6 months follow-up CF construct score, and condition (whether the participant was part of the intervention group or the control group). Continuous covariates were standardised. To assess which PA component was the more predominant factor in relation to cognitive function, both MVPA and LPA were analysed simultaneously (in the same model). Furthermore, confidence intervals (CIs) were calculated for all outcomes. Analyses were conducted on all available and valid data without any ad hoc imputation [57]. Significance levels for all analyses were set at $p<0.05$. All analyses were conducted using R [58].

\section{Results}

The seven municipalities invited a total of 14,576 inhabitants, of whom 623 provided informed consent. Thirtyeight participants withdrew from the study without completing any baseline measurements. At 6 months, 19.1\% $(112 / 585)$ of the participants who started with the study dropped out, and at 12 months this rate was 26.2\% (153/ 585). At both six and 12 months, drop-out was more likely for persons of a higher age $(6 \mathrm{~m}$ : $\mathrm{OR}=1.06,95 \%$ $\mathrm{CI}=1.01,1.12, p=0.027 ; 12 \mathrm{~m}: \mathrm{OR}=1.05,95 \% \mathrm{CI}=$ $1.00,1.10, p=0.038$ ).

As shown in Table 2, the mean age of the participants was $73.7( \pm 6.1)$ years, with $46.8 \%$ female participants. The majority of the participants were living together with a spouse $(82.1 \%)$, and $48.3 \%$ were low-educated (i.e., primary, basic vocational, or lower general school). Most participants (47.7\%) were medium impaired. The most frequent chronic illnesses that participants suffered from and that impaired PA were osteoarthritis $(51.7 \%$ of all participants), vascular diseases (44.6\%), and heart
Table 2 Baseline participant characteristics $(N=432)$

\begin{tabular}{|c|c|}
\hline Demographic characteristics & \\
\hline Age in years, mean $(S D)$ & $73.7(6.1)$ \\
\hline Gender, N (\%) & \\
\hline Male & $230(53.2 \%)$ \\
\hline Female & $202(46.8 \%)$ \\
\hline Marital status, N (\%) & \\
\hline Living single & $76(17.9 \%)$ \\
\hline Living together & $348(82.1 \%)$ \\
\hline Education, $N(\%)$ & \\
\hline Low & $202(48.3 \%)$ \\
\hline Moderate & $89(21.3 \%)$ \\
\hline High & $127(30.4 \%)$ \\
\hline Health-related characteristics & \\
\hline $\mathrm{BMI}$, median (IQR) ${ }^{\mathrm{a}}$ & $26.9(24.3-29.4)$ \\
\hline Degree of impairment, $N(\%)$ & \\
\hline Little impaired & $49(11.4 \%)$ \\
\hline Medium impaired & $205(47.7 \%)$ \\
\hline Very impaired & $176(40.9 \%)$ \\
\hline LPA in min/wk., mean (SD) & $2524(622)$ \\
\hline MVPA in min/wk., median (IQR) ${ }^{\text {a }}$ & $159(66.3-292.3)$ \\
\hline CF outcomes & \\
\hline VLT - learning curve ratio, mean (SD) & $1.85(0.55)$ \\
\hline VLT - mean no. words recalled trial $1-5$, mean (SD) & $7.24(2.08)$ \\
\hline VLT - no. words delayed recall, mean (SD) & $7.57(3.15)$ \\
\hline TMT - time B-A in sec, median (IQR) ${ }^{\text {a }}$ & $27.98(12.79-49.39)$ \\
\hline SST - SSRT in ms, mean (SD) & $176.68(98.91)$ \\
\hline LDST - no. correct subs, mean (SD) & $11.33(4.26)$ \\
\hline
\end{tabular}

Abbreviations: $S D$ Standard deviation, IQR Inter Quartile Distance, BMI Body mass index, LPA Minutes of light physical activity per week, MVPA Minutes of moderate-to-vigorous physical activity per week, CF Cognitive functioning, VLT Verbal learning test, TMT Trail making test, SST Stop-signal task, SSRT Stopsignal reaction time, LDST Letter digit substitution test. ${ }^{a}$ non-normally distributed variables 


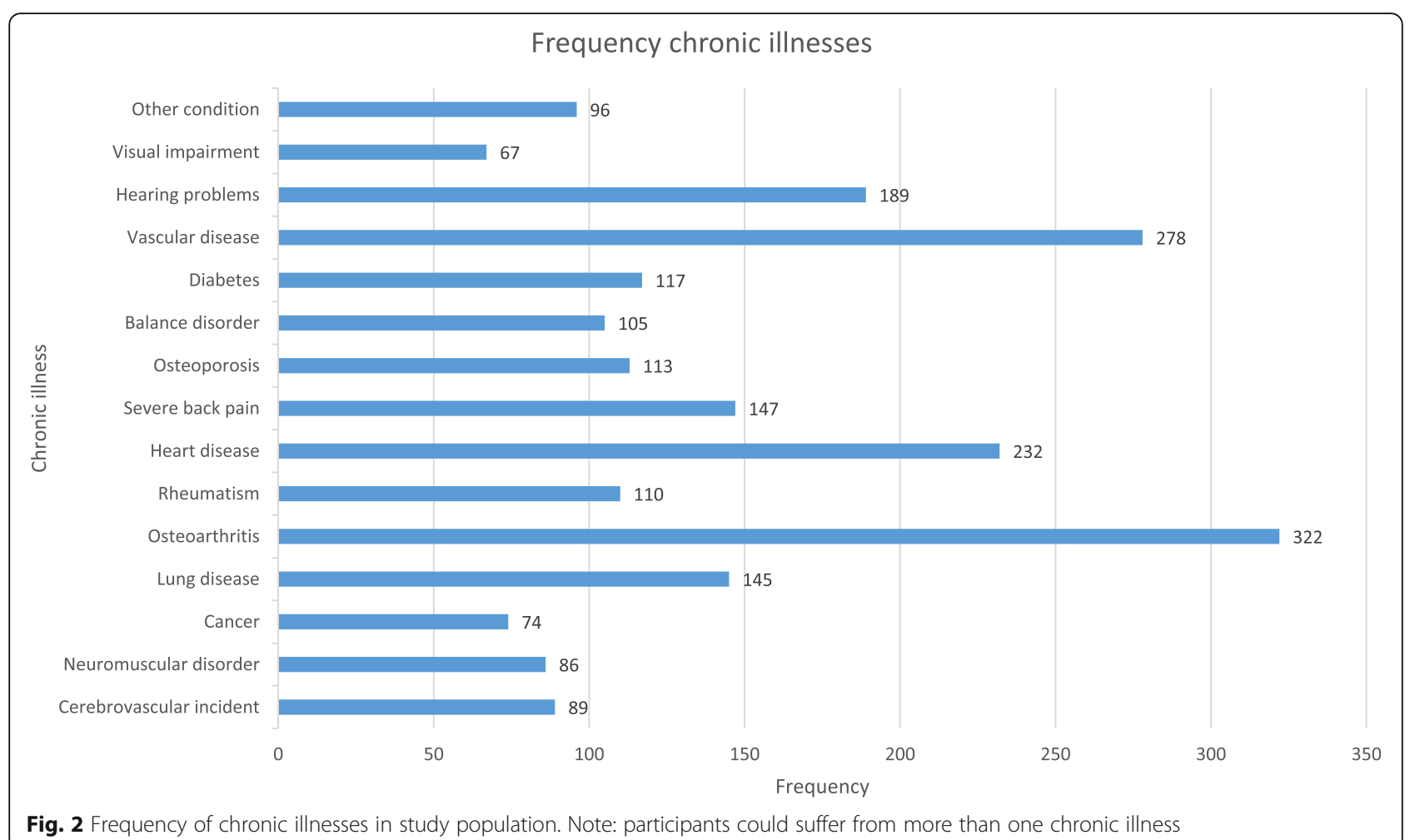

diseases (37.2\%). Participants suffered from an average of 3.5 chronic illnesses or physical impairments (Fig. 2).

\section{Associations between change in PA and change in CF over similar time periods}

Table 3 shows the results of the associations between the change in PA in the first 6 months with the change in CF outcomes over the same period. An increase in LPA between baseline and 6 months follow-up was significantly positively associated with change in the mean number of words recalled in the first five trials of the VLT (coeff. = $0.18, p \leq 0.01$ ) over the same period, showing better shortterm memory functions by an increase in LPA over time. Furthermore, an increase in LPA was significantly negatively associated with change in SSRT of the SST over the same period, indicating better inhibition scores after an increase in LPA (coeff. $=-9.84, p=0.03$ ). An increase in MVPA between baseline and 6 months was significantly negatively associated with change in the number of words recalled in the delayed recall trials of the VLT over the same period (coeff. $=-0.21, p=0.04$ ). In contrast to the results of LPA, this shows that an increase in MVPA over 6 months was associated with lower retention capacity. As there were no significant associations between the change in PA and CF over the 6-12-month period, nor between baseline and the 12-month period, these results are not displayed here but are added as supplementary files (Supplementary Tables 1 and 2). For further understanding, a

Table 3 Association between change in PA 0-6 months and change in CF over the same period ${ }^{\mathrm{b}}$

\begin{tabular}{|c|c|c|c|c|c|c|c|c|c|}
\hline \multirow[b]{2}{*}{ Change in CF 0-6 } & \multirow[b]{2}{*}{$N$} & \multicolumn{4}{|c|}{$\Delta$ LPA 0-6 } & \multicolumn{4}{|c|}{$\triangle$ MVPA 0-6 } \\
\hline & & coeff. & SE & $95 \% \mathrm{Cl}$ & $p$ & coeff. & SE & $95 \% \mathrm{Cl}$ & $p$ \\
\hline VLT - learning curve ratio & 372 & 0.02 & 0.02 & $-0.01 ; 0.06$ & 0.22 & -0.00 & 0.02 & $-0.04 ; 0.04$ & 0.88 \\
\hline VLT - mean no. words recalled trial 1-5 & 372 & 0.18 & 0.07 & $0.05 ; 0.32$ & 0.008 & -0.05 & 0.07 & $-0.19 ; 0.08$ & 0.45 \\
\hline VLT - no. words delayed recall & 373 & 0.19 & 0.10 & $-0.01 ; 0.38$ & 0.07 & -0.21 & 0.10 & $-0.41 ;-0.01$ & 0.044 \\
\hline TMT - time B-A in sec ${ }^{a}$ & 359 & 0.00 & 0.01 & $-0.02 ; 0.02$ & 0.79 & -0.01 & 0.01 & $-0.03 ; 0.02$ & 0.62 \\
\hline SST - SSRT in ms & 313 & -9.84 & 4.38 & $-18.47 ;-1.21$ & 0.026 & -0.45 & 4.51 & $-8.42 ; 9.32$ & 0.92 \\
\hline LDST - no. correct subs & 362 & -0.19 & 0.15 & $-0.49 ; 0.11$ & 0.21 & 0.18 & 0.16 & $-0.13 ; 0.49$ & 0.26 \\
\hline
\end{tabular}

Abbreviations: PA Physical activity, LPA Change in light physical activity minutes per week between 6 months follow-up and baseline, MVPA Change in moderateto-vigorous physical activity minutes per week between 6 months follow-up and baseline, SE Standard error, CI Confidence interval, ES Effect size, CF Cognitive functioning, VLT Verbal learning test, TMT Trail making test, SST Stop-signal task, SSRT Stop-signal reaction time, LDST Letter digit substitution test. ${ }^{a}$ TMT - time BA in sec was log transformed. ${ }^{b}$ Models are adjusted for baseline CF score, the covariates, and condition (control or intervention group) 
detailed description of the PA and CF outcomes at baseline, 6 months follow-up, and 12 months follow-up can be found in Supplementary Table 3.

\section{The influence of change in PA in the first six months on CF change over a year}

Table 4 shows the associations of the predictive value of change in PA in the first 6 months for change in CF outcomes between baseline and 12 months follow-up. Increased LPA between baseline and 6 months follow-up was significantly positively associated with change in the mean number of words recalled in the first five trials of the VLT test over 12 months (coeff. $=0.18, p=0.02$ ), showing that an increase in LPA is of predictive value for better short-term memory functions over an extended period. No other significant relations were found.

\section{Discussion}

The aim of this study was to investigate the longitudinal association between change in both LPA and MVPA and the change in CF in older adults with chronic illnesses. An increase in LPA in the first 6 months was significantly associated with an increase in the same timeframe in short-term verbal memory scores and improved inhibition. In addition, the change in LPA over the first 6 months was predictive for an improved short-term verbal memory after 12 months. Interestingly, an increase in MVPA in the first 6 months was significantly associated with worse longer-term verbal memory scores (lower number of words recalled during the delayed recall trial of the VLT). No significant associations were found between change in PA and change in CF aspects between baseline and 12 months follow-up and between 6 months follow-up and 12 months follow-up.

The present study established that an increase in objectively measured LPA is beneficial for some CF aspects in older adults with chronic illnesses. The results of the few past longitudinal association studies are somewhat inconsistent [9, 59-61]. A study by Hamer et al. [59] found that PA was associated with preservation of memory and executive function over 10 years follow-up. Nonetheless, PA was measured with self-report questionnaires and the authors did not make a distinction between different intensities of PA. Lee et al. [60] did evaluate the associations between LPA and cognitive decline over an eight-year period in community-dwelling adults aged 60 and older and found that LPA was associated with a slower decline in $\mathrm{CF}$ after controlling for MVPA. Yet, LPA and MVPA were assessed by interviews based on a questionnaire. Stubbs et al. [9] did use accelerometers to measure PA and found that a higher level of objectively measured LPA, independent of MVPA, was prospectively associated with better cognitive ability in community-dwelling older adults. In addition, objectively assessed MVPA was also associated with better cognitive status. Although objectively assessed PA was only measured at baseline in the study by Stubbs et al., which bars the capability to examine the relationship between changes in LPA and cognitive ability, it was one of the first longitudinal studies to find that LPA is beneficial for cognitive ability in older adults. However, cognitive ability was tested with a self-report questionnaire instead of the more objective psychological test used in our study. In contrast, Zhu et al. [61] found that a dose-response relationship exists between engagement in MVPA and cognitive performance, tested with neuropsychological tests, over time. Yet, no relationship between LPA and cognitive performance over time was found. Notwithstanding, this study also only assessed PA at baseline, contrary to our study. It is suggested that this field of research needs more studies that objectively measure their outcomes.

A possible explanation for the finding that only an increase in LPA was positively associated with an improvement in CF and that an increase in MVPA was even negatively associated with change in one CF outcome can be found in the type of activities belonging to LPA and MVPA. Typical LPA activities are casually walking

Table 4 Association between change in PA 0-6 months and change in CF 0-12 months ${ }^{b}$

\begin{tabular}{|c|c|c|c|c|c|c|c|c|c|}
\hline \multirow[b]{2}{*}{ Change in CF 0-12 } & \multirow[b]{2}{*}{$N$} & \multicolumn{4}{|c|}{$\Delta$ LPA 0-6 } & \multicolumn{4}{|c|}{$\triangle$ MVPA 0-6 } \\
\hline & & coeff. & SE & $95 \% \mathrm{Cl}$ & $p$ & coeff. & SE & $95 \% \mathrm{Cl}$ & $p$ \\
\hline VLT - learning curve ratio & 372 & 0.03 & 0.02 & $-0.01 ; 0.06$ & 0.18 & -0.02 & 0.02 & $-0.06 ; 0.02$ & 0.33 \\
\hline VLT - mean no. words recalled trial $1-5$ & 372 & 0.18 & 0.07 & $0.03 ; 0.33$ & 0.016 & -0.08 & 0.07 & $-0.23 ; 0.07$ & 0.32 \\
\hline VLT - no. words delayed recall & 374 & 0.20 & 0.11 & $-0.02 ; 0.42$ & 0.08 & -0.00 & 0.11 & $-0.23 ; 0.22$ & 0.97 \\
\hline TMT - time B-A in sec ${ }^{a}$ & 360 & -0.01 & 0.01 & $-0.03 ; 0.01$ & 0.25 & -0.00 & 0.01 & $-0.02 ; 0.02$ & 0.91 \\
\hline SST - SSRT in ms & 306 & 0.98 & 4.28 & $-7.44 ;-9.40$ & 0.82 & -6.76 & 4.36 & $-15.35 ; 1.82$ & 0.12 \\
\hline LDST - no. correct subs & 350 & 0.12 & 0.17 & $-0.20 ; 0.45$ & 0.46 & -0.13 & 0.17 & $-0.47 ; 0.20$ & 0.44 \\
\hline
\end{tabular}

Abbreviations: PA Physical activity, LPA Change in light physical activity minutes per week between six months follow-up and baseline, MVPA change in moderateto-vigorous physical activity minutes per week between six months follow-up and baseline, SE Standard error, CI Confidence interval, ES Effect size, CF Cognitive functioning, VLT Verbal learning test, TMT Trail making test, SST Stop-signal task, SSRT Stop-signal reaction time, LDST Letter digit substitution test. ${ }^{a}$ TMT - time BA in sec was log transformed. ${ }^{b}$ Models are adjusted for baseline CF score, the covariates, and condition (control or intervention group) 
and household chores. Conceivably, these activities offer more opportunity for cognitive engagement with other people, listening to music, or enjoying the outdoors, and it is known that social and intellectual activities of daily life are associated with higher cognitive performance $[62,63]$. Common MVPA activities, such as brisk walking or bicycling, are possibly too exhausting for further cognitive engagement, especially for older adults with chronic illnesses. However, a recent study suggests that MVPA activities that do require greater cognitive engagement, such as dancing and exercise class aerobics, do lead to greater training effects on cognition and brain connectivity than exercise requiring lower cognitive loads, such as walking briskly, in healthy elderly people [64]. Nonetheless, dancing and exercise class aerobics are generally less often executed by older adults with chronic illnesses $[65,66]$.

Next to cognitive aspects related to physical activities, another possible explanation could be the assumed underlying mechanisms that are responsible for changing CF through PA. A study by Voelcker-Rehage et al. [29] shows that besides cardiovascular training, other types of PA (i.e. coordination training) also improve $\mathrm{CF}$ of older adults. However, the mechanisms that result in these changes seem to differ depending on the intervention. Cardiovascular training was associated with increased activation of the sensorimotor network, whereas coordination training was associated with increased activation in the visual-spatial network. These differences in affected aspects of CF were also found in a recent study in rodents by Vilela et al. [67]. They showed that aerobic exercise and resistance training improved spatial working memory and hippocampal plasticity in ageing rats. However, they found that different molecular mechanisms were responsible for this. While both interventions increased neurotrophic signalling, aerobic exercise increased glutamatergic signalling and reduced DNA damage, and resistance training increased proinflammatory factors. To conclude, differences in type of PA (providing opportunity for cognitive engagement or not) and underlying molecular and neurological mechanisms related to changes in CF can explain why some aspects of CF have improved over time due to increased PA and some CF aspects did not. However, this seems too little to "justify" why there was a negative association between MVPA and long-term memory. It is clear that more research is needed in this field to affirm our findings and our possible explanation for the results.

Our findings are important in the context of the suitableness of the prescription of LPA to older adults with chronic illnesses. This population often has limited mobility and suffers from pain and fatigue [31]. As a result, older adults with chronic illnesses may be deconditioned or are not used to exercise and thus restricted to LPA only. LPA activities such as casual walking, gardening, and household chores are preferred PA activities for older adults [66, 68]. Moreover, LPA may also offer opportunities to interact with other people and thus reduce the risk of social isolation. Furthermore, increasing PA in general, especially through MVPA, is often challenging and sometimes comes along with risks of injury and deterioration as a result of physical complications. Although the PA guidelines [14] prescribe a minimum of $150 \mathrm{~min}$ of MVPA per week for older adults with chronic illnesses, such as type-2 diabetes, hypertension, HIV and cancer survivors, some chronic illnesses have contraindications [69] for MVPA (i.e., recent myocardial infarction, complete heart block, acute congestive heart failure, unstable angina, and uncontrolled hypertension). Increasing LPA is probably easier and, in some cases, safer for older adults [8]. Furthermore, it is probably easier to maintain in the long term. If more research confirms the results of this study, it is warranted to prescribe LPA next to MVPA for older adults with chronic illnesses to gain both health benefits and cognitive benefits. In this case, future interventions, PA guidelines, and PA programmes should address this finding.

This study has several strengths. Firstly, we objectively assessed PA with accelerometers. Although they have limitations in distinguishing between types of activities, they are considered a better measurement tool for PA than self-report questionnaires [70]. These questionnaires are prone to over-reporting and have issues with validly assessing LPA [10, 71]. Secondly, our research population is reasonably representative of the general older adult population in the Netherlands, as almost equal groups of males and females participated and most of the participants were low educated (e.g., 51\%) [72]. Furthermore, BMI levels and the mean number of comorbidities (3.5) are also comparable to the general older adult population of the Netherlands [73, 74]. Therefore, these results could be generalisable to the older adults with chronic illnesses population or even to the general older adult population of the Netherlands.

This study also has some limitations. First, this study only tested the associations of change in LPA and MVPA with change in CF. To test the actual effects of change in PA on $\mathrm{CF}$, a randomised controlled trial must be carried out with at least three groups (LPA intervention, MVPA intervention, control group). As our own Active Plus intervention was mainly aimed at stimulating MVPA, and we only included one experimental group in the randomised controlled trial [40], we could not test the intervention effects of change in LPA and MVPA on CF separately. In addition, isolating the independent contribution of both PA intensities is difficult. Also, it would be of great interest to include sedentary behaviour in future analyses, as it becomes more and more clear 
that excessive sedentary behaviour has detrimental effects on physical health [75]. Furthermore, the study period of 1 year was quite short. Future longitudinal research of longer duration is required to verify our oneyear findings. Another limitation was that we performed multiple tests to analyse the associations of PA with CF. This gives a broader perspective on $\mathrm{CF}$ functioning instead of assessing one specific test. However, the more tests done, the more likely faulty conclusions are drawn, because the probability of a type 1 error is increased [76]. A Bonferroni correction, however, assumes that all of the hypothesis tests are statistically independent, which is not the case in the current study, as these aspects of CF are dependent. Therefore, a Bonferroni correction would be overly conservative. However, the results of this study should be considered with caution.

\section{Conclusions}

An increase in LPA in the first 6 months was associated with better short-term verbal memory and inhibition over the same period. Furthermore, an increase in LPA in the first 6 months was a predictive value for change in short-term verbal memory over a 12 -month period. MVPA, however, at the first 6 months was associated with worse longer-term verbal memory scores. It may be that LPA activities offered opportunities for PA with greater cognitive engagement than MVPA activities or that the underlying molecular and neurological mechanisms related to changes in CF differ per PA type. Thus, for older adults with chronic illnesses who may experience difficulties in being sufficiently active, an increase in LPA is probably more achievable than an increase in MVPA. In addition, an increase in LPA enhances CF more than an increase in MVPA does.

\section{Abbreviations \\ BMI: Body mass index; cETO: Research Ethics Committee of the Open University; Cl: Confidence intervals; CF: Cognitive functioning; ES: Effect size; ICC: Intra cluster correlation; IQR: Inter quartile distance; LDST: Letter digit substitution test; LPA: Light physical activity; MMSE: Mini mental state examination; MVPA: Moderate-to-vigorous physical activity; OR: Odds-ratio; PA: Physical activity; SD: Standard deviation; SE: Standard error; SSRT: Stop- signal reaction time; SST: Stop-signal task; TMT: Trail making test; VLT: Verbal learning test}

\section{Supplementary Information}

The online version contains supplementary material available at https://doi. org/10.1186/s12877-021-02429-x.

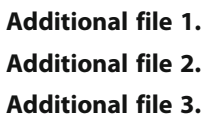

\section{Acknowledgments}

We want to thank OverNite Software Europe for their support in developing and executing the online intervention. We also want to thank the municipalities of Heerlen, Maastricht, Landgraaf, Leudal, Nederweert, Venlo and De Bilt for sending invitations to their inhabitants. In addition, we would like to thank all participants, research assistants Audrey Beaulen and Mara Kirschner, and students who helped conducting the study.

\section{Authors' contributions}

Conceptualization: EV, CB, RG, LL; Data curation: EV; Formal analysis: EV; Funding acquisition: $\mathrm{CB}, \mathrm{RG}$, LL; Investigation: $\mathrm{EV}$; Methodology, EV, CB, RG, LL; Project administration: EV; Supervision: CB, RG, LL; Writing - original draft: EV; Writing - review and editing: CB, RG, LL. The author(s) read and approved the final manuscript.

\section{Funding}

This project was funded by Brain Foundation Netherlands ('Hersenstichting') embedded in the program "Physical Activity and Cognition (GH-2016-03-01). The Brain Foundation peer reviewed the design of this study. This funding source had no role during its execution, analyses, interpretation of the data, or decision to submit results.

Availability of data and materials

Study data are available from the corresponding author on reasonable request.

\section{Declarations}

\section{Ethics approval and consent to participate}

Ethical approval for the study was obtained from the Research Ethics Committee (CETO) of the Open University and the trial is registered with the Dutch Trial Register, protocol number NL6005. All participants have provide written informed consent prior to commencing the study. The study was conducted following the Declaration of Helsinki.

\section{Consent for publication}

Not applicable.

\section{Competing interests}

The authors declare that they have no competing interests.

\section{Author details}

${ }^{1}$ Faculty of Psychology, Open University of the Netherlands, 6419 AT Heerlen, the Netherlands. ${ }^{2}$ Faculty of Educational Sciences, Open University of the Netherlands, 6419 AT Heerlen, the Netherlands. ${ }^{3}$ Nutrition and Translational Research in Metabolism (School NUTRIM), Maastricht University, 6200 MD Maastricht, the Netherlands.

Received: 22 February 2021 Accepted: 21 July 2021

Published online: 04 September 2021

\section{References}

1. Bárrios H, Narciso S, Guerreiro M, Maroco J, Logsdon R, de Mendonça A Quality of life in patients with mild cognitive impairment. Aging Ment Health. 2013;17(3):287-92. https://doi.org/10.1080/13607863.2012.747083.

2. Kim JS, Park E, An M. The cognitive impact of chronic diseases on functional capacity in community-dwelling adults. J Nurs Res. 2019;27(1):e3-8. https:// doi.org/10.1097/jnr.0000000000000272.

3. Gajewski PD, Falkenstein M. Physical activity and neurocognitive functioning in aging - a condensed updated review. Eur Rev Aging and Phys Act. 2016; 13(1). https://doi.org/10.1186/s11556-016-0161-3.

4. Northey JM, Cherbuin N, Pumpa KL, Smee DJ, Rattray B. Exercise interventions for cognitive function in adults older than 50: a systematic review with meta-analysis. Br J Sports Med. 2018;52(3):154-60. https://doi. org/10.1136/bjsports-2016-096587.

5. Macpherson H, Teo WP, Schneider LA, Smith AE. A life-long approach to physical activity for brain health. Front Aging Neurosci. 2017;9:147. https:// doi.org/10.3389/fnagi.2017.00147.

6. Bullard T, Ji M, An R, Trinh L, MacKenzie M, Mullen SP. A systematic review and meta-analysis of adherence to physical activity interventions among three chronic conditions: Cancer, cardiovascular disease, and diabetes. BMC Public Health. 2019;19(1):636. https://doi.org/10.1186/s12889-019-6877-z.

7. Umegaki H, Makino T, Uemura K, Shimada H, Cheng XW, Kuzuya M. Objectively measured physical activity and cognitive function in urbandwelling older adults. Geriatr Gerontol Int. 2018;18(6):922-8. https://doi. org/10.1111/ggi.13284 
8. Johnson LG, Butson ML, Polman RC, Raj IS, Borkoles E, Scott D, et al. Light physical activity is positively associated with cognitive performance in older community dwelling adults. J Sci Med Sport. 2016;19(11):877-82. https://doi. org/10.1016/j.jsams.2016.02.002.

9. Stubbs B, Chen LJ, Chang CY, Sun WJ, Ku PW. Accelerometer-assessed light physical activity is protective of future cognitive ability: a longitudinal study among community dwelling older adults. Exp Gerontol. 2017;91:104-9. https://doi.org/10.1016/j.exger.2017.03.003.

10. Skender S, Ose J, Chang-Claude J, Paskow M, Brühmann B, Siegel EM, et al. Accelerometry and physical activity questionnaires - a systematic review. BMC Public Health. 2016;16(1):515. https://doi.org/10.1186/s12889-016-3172-0.

11. Piercy KL, Troiano RP, Ballard RM, Carlson SA, Fulton JE, Galuska DA, et al. The physical activity guidelines for Americans. JAMA. 2018;320(19):2020-8. https://doi.org/10.1001/jama.2018.14854.

12. Amagasa S, Machida M, Fukushima N, Kikuchi H, Takamiya T, Odagiri Y, et al. Is objectively measured light-intensity physical activity associated with health outcomes after adjustment for moderate-to-vigorous physical activity in adults? A systematic review. Int J Behav Nutr Phys Act. 2018;15(1):65. https://doi.org/10.1186/s1 2966-018-0695-z.

13. Haskell WL, Lee IM, Pate RR, Powell KE, Blair SN, Franklin BA, et al. Physical activity and public health: updated recommendation for adults from the American College of Sports Medicine and the American Heart Association. Circulation. 2007;116(9):1081-93. https://doi.org/10.1161/CIRCULATIONAHA.1 07.185649 .

14. WHO guidelines on physical activity and sedentary behaviour. Geneva: World Health Organization; 2020.

15. Warburton DER, Nicol CW, Bredin SSD. Health benefits of physical activity: the evidence. CMAJ. 2006;174(6):801-9. https://doi.org/10.1503/cmai.051351.

16. Füzéki E, Engeroff T, Banzer W. Health benefits of light-intensity physical activity: a systematic review of accelerometer data of the national health and nutrition examination survey (NHANES). Sports Med. 2017;47(9):176993. https://doi.org/10.1007/s40279-017-0724-0.

17. Logsdon RG, Hochhalter AK, Sharkey JR. From message to motivation: where the rubber meets the road. Gerontologist. 2009;49(S1):S108-11. https://doi.org/10.1093/geront/gnp074.

18. Colcombe S, Kramer AF. Fitness effects on the cognitive function of older adults: a meta-analytic study. Psychol Sci. 2003;14(2):125-30. https://doi. org/10.1111/1467-9280.t01-1-01430.

19. Kirk-Sanchez NJ, McGough EL. Physical exercise and cognitive performance in the elderly: current perspectives. Clin Interv Aging. 2014;9:51-62. https:// doi.org/10.2147/CIA.S39506.

20. Bherer L, Erickson KI, Liu-Ambrose T. A review of the effects of physical activity and exercise on cognitive and brain functions in older adults. J Aging Res. 2013;657508:1-8. https://doi.org/10.1155/2013/657508.

21. Kelly ME, Loughrey D, Lawlor BA, Robertson IH, Walsh C, Brennan S. The impact of exercise on the cognitive functioning of healthy older adults: a systematic review and meta-analysis. Ageing Res Rev. 2014;16:12-31. https:// doi.org/10.1016/j.arr.2014.05.002.

22. Smith PJ, Blumenthal JA, Hoffman BM, Cooper H, Strauman TA, WelshBohmer $\mathrm{K}$, et al. Aerobic exercise and neurocognitive performance: a metaanalytic review of randomized controlled trials. Psychosom Med. 2010;72(3): 239-52. https://doi.org/10.1097/PSY.0b013e3181d14633.

23. Erickson Kl, Hillman C, Stillman CM, Ballard RM, Bloodgood B, Conroy DE, et al. Physical activity, cognition, and brain outcomes: a review of the 2018 physical activity quidelines. Med Sci Sports and Exerc. 2019;51(6):1242-51. https://doi.org/10.1249/MSS.0000000000001936.

24. Snowden M, Steinman L, Mochan K, Grodstein F, Prohaska TR, Thurman DJ, et al. Effect of exercise on cognitive performance in community-dwelling older adults: review of intervention trials and recommendations for public health practice and research. J Am Geriatr Soc. 2011;59(4):704-16. https:// doi.org/10.1111/j.1532-5415.2011.03323.x.

25. Van Uffelen JGZ, Chin A, Paw MJM, Hopman-Rock M, Van Mechelen W. The effects of exercise on cognition in older adults with and without cognitive decline: a systematic review. Clin J Sport Med. 2008;18(6):486-500. https:// doi.org/10.1097/JSM.0b013e3181845fob.

26. Young J, Angevaren M, Rusted J, Tabet N. Aerobic exercise to improve cognitive function in older people without known cognitive impairment. Cochrane Database Syst Rev. 2015;(4):CD005381. https://doi.org/10.1002/14 651858.CD005381.pub4.

27. Frost NJ, Weinborn M, Gignac GE, Rainey-Smith SR, Markovic S, Gordon N, et al. A randomized controlled trial of high-intensity exercise and executive functioning in cognitively normal older adults. Am J Geriatr Psychiatry. 2021, 29(2):129-40. https://doi.org/10.1016/j.jagp.2020.06.015.

28. Gomes-Osman J, Cabral DF, Morris TP, Mclnerney K, Cahalin LP, Rundek T, et al. Exercise for cognitive brain health in aging: a systematic review for an evaluation of dose. Neurol Clin Pract. 2018;8(3):257-65. https://doi.org/1 0.1212/CPJ.00000000000000460.

29. Voelcker-Rehage C, Godde B, Staudinger UM. Cardiovascular and coordination training differentially improve cognitive performance and neural processing in older adults. Front Hum Neurosci. 2011;5:26. https://doi org/10.3389/fnhum.2011.00026.

30. Niemann C, Godde B, Staudinger UM, Voelcker-Rehage C. Exercise-induced changes in basal ganglia volume and cognition in older adults. Neuroscience. 2014;281:147-63. https://doi.org/10.1016/j.neuroscience.2014. 09.033.

31. Fuller BG, Stewart Williams JA, Byles JE. Active living-the perception of older people with chronic conditions. Chronic IIIn. 2010;6(4):294-305. https://doi. org/10.1177/1742395310377673.

32. Tak E, Kuiper R, Chorus A, Hopman-Rock M. Prevention of onset and progression of basic $A D L$ disability by physical activity in community dwelling older adults: a meta-analysis. Ageing Res Rev. 2013;12(1):329-38. https://doi.org/10.1016/j.arr.2012.10.001.

33. Volders E, De Groot RHM, Coumans JMJ, Bolman CAW, Lechner L. A randomized controlled trial into the cognitive effects of a computer-tailored physical activity intervention in older adults with chronic disease(s). Eur Rev Aging Phys Act. 2021;18(1):1-15. https://doi.org/10.1186/s11556-021-00259-9.

34. Volders E, Bolman CAW, De Groot RHM, Verboon P, Lechner L. The effect of active plus, a computer-tailored physical activity intervention, on the physical activity of older adults with chronic illness (es)-a cluster randomized controlled trial. Int J Environ Res Public Health. 2020;17(7):2590. https://doi.org/10.3390/ijerph17072590.

35. Thomas AG, Dennis A, Bandettini PA, Johansen-Berg H. The effects of aerobic activity on brain structure. Front Psychol. 2012;3:86. https://doi.org/1 0.3389/fpsyg.2012.00086

36. Murman DL. The impact of age on cognition. Semin Hear. 2015;36(3):11121. https://doi.org/10.1055/s-0035-1555115.

37. Van der Elst W, Van Boxtel MPJ, Van Breukelen GJP, Jolles J. Rey's verbal learning test: normative data for 1855 healthy participants aged 24-81 years and the influence of age, sex, education, and mode of presentation. J Int Neuropsychol Soc. 2005;11(3):290-302. https://doi.org/10.1017/S135561 7705050344.

38. Van Der Elst W, Van Boxtel M, Van Breukelen G, Jolles J. The letter digit substitution test: normative data for 1,858 healthy participants aged 24-81 from the Maastricht aging study (MAAS): influence of age, education, and sex. J Clin Exp Neuropsychol. 2006;28(6):998-1009. https://doi.org/10.1 080/13803390591004428

39. Miyake A, Friedman NP, Emerson MJ, Witzki AH, Howerter A, Wager TD. The unity and diversity of executive functions and their contributions to complex "frontal lobe" tasks: a latent variable analysis. Cogn Psychol. 2000; 41(1):49-100. https://doi.org/10.1006/cogp.1999.0734.

40. Volders E, Bolman CAW, De Groot RHM, Lechner L. The effect of active plus, a computer-tailored physical activity intervention, on cognitive functioning of elderly people with chronic illness (es) - study protocol for a randomized controlled trial. BMC Public Health. 2019;19(1):1197. https://doi.org/10.1186/ \$12889-019-7517-3.

41. Drain SC. Inquisit 5. Seattle: Millisecond Software; 2016. Available from: https://www.millisecond.com

42. De Jong R, Coles MGH, Logan GD, Gratton G. In search of the point of no return: the control of response processes. J Exp Psy Hum Percept Perform. 1990;16(1):164-82. https://doi.org/10.1037/0096-1523.16.1.164.

43. Tannock R, Schachar RJ, Carr RP, Chajczyk D, Logan GD. Effects of methylphenidate on inhibitory control in hyperactive children. J Abnorm Child Psychol. 1989;17(5):473-91. https://doi.org/10.1007/BF00916508.

44. Congdon E, Mumford JA, Cohen JR, Galvan A, Canli T, Poldrack RA. Measurement and reliability of response inhibition. Front Psychol. 2012; 3(37). https://doi.org/10.3389/fpsyg.2012.00037.

45. Gorman E, Hanson HM, Yang PH, Khan KM, Liu-Ambrose T, Ashe MC. Accelerometry analysis of physical activity and sedentary behavior in older adults: a systematic review and data analysis. Eur Rev Aging Phys Act. 2014; 11(1):35-49. https://doi.org/10.1007/s11556-013-0132-x.

46. Migueles JH, Cadenas-Sanchez C, Ekelund U, Delisle Nyström C, MoraGonzalez J, Löf M, et al. Accelerometer data collection and processing 
criteria to assess physical activity and other outcomes: a systematic review and practical considerations. Sport Med. 2017;47(9):1821-45. https://doi. org/10.1007/s40279-017-0716-0.

47. Choi L, Ward SC, Schnelle JF, Buchowski MS. Assessment of wear/nonwear time classification algorithms for triaxial accelerometer. Med Sci Sports Exerc. 2012:44(10):2009-16. https://doi.org/10.1249/MSS.0b013e318258cb36.

48. Sasaki JE, John D, Freedson PS. Validation and comparison of ActiGraph activity monitors. J Sci Med Sport. 2011;14(5):411-6. https://doi.org/10.1016/ j.jsams.2011.04.003.

49. Aguilar-Farías N, Brown WJ, Peeters GMEE. ActiGraph GT3X+ cut-points for identifying sedentary behaviour in older adults in free-living environments. $J$ Sci Med Sport. 2014;17(3):293-9. https://doi.org/10.1016/j.jsams.2013.07.002.

50. Rey A. L'examen clinique en psychologie, vol. 222. Oxford: Presses Universitaries De France; 1958.

51. Reitan RM, Wolfson D. The Halstead-Reitan neuropsychological test battery: theory and clinical interpretation (2nd ed.). Tucson, AZ: neuropsychology press. Tucson: Neuropsychological Press; 1993.

52. Corrigan JD, Hinkeldey NS. Relationships between parts a and B of the trail making test. J Clin Psychol. 1987;43(4):402-9. https://doi.org/10.1002/1097-4 679(198707)43:4<402::AID-JCLP2270430411>3.0.CO;2-E.

53. Verbruggen F, Logan GD, Stevens MA. STOP-IT: windows executable software for the stop-signal paradigm. Behav Res Methods. 2008;40(2):47983. https://doi.org/10.3758/BRM.40.2.479.

54. Lawrence LM, Singleton JF. What do we mean by older adult and physical activity? Reviewing the use of these terms in recent research. Act Adapt Aging. 2017;41(1):22-46. https://doi.org/10.1080/01924788.2016.1272391.

55. Depp CA, Jeste DV. Definitions and predictors of successful aging: a comprehensive review of larger quantitative studies. Am I Geriatr Psychiatry. 2006;14(1):6-20. https://doi.org/10.1097/01.JGP.0000192501.03069.bc.

56. Boekhout JM, Peels DA, Berendsen BAJ, Bolman CAW, Lechner L. An eHealth intervention to promote physical activity and social network of single, chronically impaired older adults: adaptation of an existing intervention using intervention mapping. JMIR Res Protoc. 2017;6(11):e230. https://doi.org/10.2196/resprot.8093.

57. Twisk JWR. Applied multilevel analysis: a practical guide for medical researchers. Cambridge: Cambridge University Press; 2006. https://doi.org/1 $0.1017 /$ CBO9780511610806

58. $\mathrm{R}$ Core Team. A language and environment for statistical computing. Vienna: R Foundation for Statistical Computing; 2019. Available from: https://www.R-project.org

59. Hamer M, Terrera GM, Demakakos P. Physical activity and trajectories in cognitive function: English longitudinal study of ageing. J Epidemiol Community Health. 2018; 72(6):477-83. https:/doi.org/10.1136/jech-2017-210228.

60. Lee S, Yuki A, Nishita Y, Tange C, Kim H, Kozakai R, et al. Research relationship between light-intensity physical activity and cognitive function in a community-dwelling elderly population-An 8-year longitudinal study. J Am Geriatr Soc. 2013;61(3):452-3. https://doi.org/10.1111/jgs.12119.

61. Zhu W, Wadley VG, Howard VJ, Hutto B, Blair SN, Hooker SP. Objectively measured physical activity and cognitive function in older adults. Med Sci Sports Exerc. 2017;49(1):47-53. https://doi.org/10.1249/MSS.0000000000001079.

62. Sposito G, Neri AL, Yassuda MS. Cognitive performance and engagement in physical, social and intellectual activities in older adults: the FIBRA study. Dement Neuropsychol. 2015;9(3):270-8. https://doi.org/10.1590/1980-57642 015DN93000010

63. Dause T, Kirby E. Aging gracefully: social engagement joins exercise and enrichment as a key lifestyle factor in resistance to age-related cognitive decline. Neural Regen Res. 2019;14(1):39-42. https://doi.org/10.4103/1673-53 74.243698 .

64. Chao YP, Wu CW, Lin LJ, Lai CH, Wu HY, Hsu AL, et al. Cognitive load of exercise influences cognition and neuroplasticity of healthy elderly: An exploratory investigation. J Med Biol Eng. 2020;40(3):391-9. https://doi.org/1 0.1007/s40846-020-00522-x.

65. Van den Dool R. Deelname aan sport en bewegen door ouderen. Utrecht: Mulier Instituut; 2019.

66. Ashe MC, Miller WC, Eng JJ, Noreau L. Older adults, chronic disease and leisure-time physical activity. Gerontology. 2009:55(1):64-72. https://doi. org/10.1159/000141518.

67. Vilela TC, Muller AP, Damiani AP, Macan TP, da Silva S, Canteiro PB, et al. Strength and aerobic exercises improve spatial memory in aging rats through stimulating distinct neuroplasticity mechanisms. Mol Neurobiol. 2017 Dec;54(10):7928-37. https://doi.org/10.1007/s12035-016-0272-x.
68. Farren L, Belza B, Allen P, Brolliar S, Brown DR, Cormier ML, et al. Mall walking program environments, features, and participants: a scoping review. Prev Chronic Dis. 2015;12:e129. https://doi.org/10.5888/pcd12.150027.

69. Elsawy B, Higgins KE. Physical activity guidelines for older adults. Am Fam Physician. 2010 Jan 1;81(1):55-9.

70. Arvidsson D, Fridolfsson J, Börjesson M. Measurement of physical activity in clinical practice using accelerometers. J Intern Med. 2019;286(2):137-53. https://doi.org/10.1111/joim.12908.

71. Shephard RJ. Limits to the measurement of habitual physical activity by questionnaires. Br J Sports Med. 2003;37(3):197-206. https://doi.org/10.1136/ bjsm.37.3.197.

72. CBS. StatLine - Bevolking; hoogstbehaald onderwijsniveau en onderwijsrichting. Available from: https://opendata.cbs.nl/statline/\#/CBS/nl/ dataset/82816ned/table?dl=8083 [cited 2020 Dec 10].

73. CBS. StatLine - Leefstijl en (preventief) gezondheidsonderzoek; persoonskenmerken Available from: https://opendata.cbs.nl/statline/\#/CBS/ nl/dataset/83021 NED/table?ts=1522312658353 [cited 2020 Feb 19].

74. Volksgezondheidenzorg.info. Chronische ziekten en multimorbiditeit-Cijfers \& Context-Huidige situatie. [Internet]. RIVM: Bilthoven. 2016 [cited 2019 Feb 5]. DOI: https://doi.org/10.1186/s12942-017-0097-5, 16, 1, 23.

75. Young DR, Hivert MF, Alhassan S, Camhi SM, Ferguson JF, Katzmarzyk PT, et al. Sedentary behavior and cardiovascular morbidity and mortality: a science advisory from the American Heart Association. Circulation. $2016 \mathrm{Sep}$ 27;134(13):e262-79. https://doi.org/10.1161/CIR.0000000000000440.

76. Chen SY, Feng Z, Yi X. A general introduction to adjustment for multiple comparisons. J Thorac Dis. 2017;9(6):1725-9. https://doi.org/10.21037/jtd.201 7.05.34.

\section{Publisher's Note}

Springer Nature remains neutral with regard to jurisdictional claims in published maps and institutional affiliations.
Ready to submit your research? Choose BMC and benefit from:
- fast, convenient online submission
- thorough peer review by experienced researchers in your field
- rapid publication on acceptance
- support for research data, including large and complex data types
- gold Open Access which fosters wider collaboration and increased citations
- maximum visibility for your research: over $100 \mathrm{M}$ website views per year
At BMC, research is always in progress.
Learn more biomedcentral.com/submissions 